\title{
Depth Dependence of Nanoindentation Pile-Up Patterns in Copper Single Crystals
}

\author{
STANISŁAW KUCHARSKI and DARIUSZ JARZĄBEK
}

\begin{abstract}
A study of the dependence of nanoindentation pile-up patterns on the indentation load and crystallographic orientation is presented. Three different orientations-(001), (011), and (111) - of single crystal copper were investigated. Experiments were conducted on a CSM ultrananoindentation tester using a Berkovich tip. The topographic images were obtained using an atomic force microscope. The evolution of pile-up patterns with different applied loads was observed. The results show that for applied loads equal to $0.45 \mathrm{mN}$ and smaller the pile-up patterns do not depend on the crystallographic orientation of the indented surface; instead, they depend on the tip's geometry. On the other hand, in the case of indentation loads bigger than $2 \mathrm{mN}$, pile-up patterns on the surfaces of (001)-, (011)-, and (111)-oriented single crystals have fourfold, twofold, and sixfold (or threefold) symmetry, respectively. An intermediate state was also reported. Furthermore, a detailed analysis of residual impressions with maximal applied loads equal to $2 \mathrm{mN}$ and bigger reveals that both pile-up and sink-in patterns are present.
\end{abstract}

DOI: $10.1007 / \mathrm{s} 11661-014-2437-4$

(C) The Author(s) 2014. This article is published with open access at Springerlink.com

\section{INTRODUCTION}

NanOINDENTAtion is a technique which has been used recently to determine the mechanical properties and characterizes the mechanical behavior of materials and thin films at nano- and micro-scales. An important feature of these experiments is the deformation of the material around the contact area: sinking-in (deformation downward with respect to the indented surface plane) and piling-up (deformation upward). For isotropic materials, the generation of pile-ups and sinkins in indentation tests is correlated with material properties. ${ }^{[1]}$ Maximum pile-up is observed in the case of elastic-perfectly plastic materials, where strain hardening does not occur. The maximum sink-in appears when the indented material exhibits a strong strain hardening. For a specific material, with moderate strain hardening there is neither pile-up nor sink-in, and then the boundary of contact can be determined as a crosssection of the tip under loading and a surface of the sample prior to the deformation. The symmetry of pileup corresponds to the symmetry of the applied indenter tip; an axis-symmetric tip generates axis-symmetrical pile-up, and three and four pile-ups occur as a result of Berkovich and Vickers indentation, respectively. The mode of material deformation in the vicinity of a contact boundary, that is, formation of a pile-up or a sink-in, depends uniquely on the material properties and not on the applied load. Therefore, pile-up and sink-in patterns can provide useful information about the mechanical behavior of the investigated material.

STANISŁAW KUCHARSKI, Head, and DARIUSZ JARZABEK, Assistant, are with the Surface Layer Laboratory, Institute of Fundamental Technological Research, Pawińskiego 5b, 02-106 Warsaw, Poland. Contact e-mail: skuchar@ippt.pan.pl

Manuscript submitted April 3, 2014.

Article published online July 10, 2014
Nevertheless, in the case of anisotropic material, the response around the impression is more complex. Hence, much research in recent years has focused on the anisotropy associated with nanoindentation tests of a single crystal in different crystallographic orientations. Some initial attempts focusing on this topic can be found in the work of Vangroenou and Kadijk. ${ }^{[2]}$ More recently, Wang et $a l^{[3]}$ presented a study of the dependence of nanoindentation pile-up patterns and microtextures on the crystallographic orientation using high purity copper single crystals. Their results showed that the pile-up patterns on the surfaces of (001)-, (011)-, and (111)-oriented single crystals have four-, two-, and six-fold symmetry, respectively. They evaluated the crystallographic directions in which the pile-ups should appear for specific oriented surfaces and they observed that at ambient temperature the active glide systems of copper consist of (111) glide planes and $\langle 110\rangle$ slip directions. A similar effect was also observed by Liu et $a l^{[4]}$ and was compared with finite element simulations. However, in this work, threefold instead of sixfold symmetry was observed in the case of (111)-oriented surfaces. A detailed 3D study of the microstructure and texture below a conical nanoindent in a (111) $\mathrm{Cu}$ single crystal at nanometer-scale resolution was carried out by Zaafarani et al. ${ }^{[5,6]}$ These experiments revealed pronounced deformation-induced $3 \mathrm{D}$ patterning of the lattice rotations below and around the indent. On the other hand, the crystal plasticity finite element method (CPFEM) has been used to describe the phenomenon of nanoindentation pile-up patterns. This approach was presented by Casals et al. ${ }^{[7,8]}$ In their first mentioned paper, CPFEM simulations of pyramidal indentation (both Vickers and Berkovich indenters) in copper single crystals were compared with experimental results. In the second one, CPFEM was used to investigate the anisotropy in the contact response of fcc and hcp single 
crystals. To this aim, spherical indentation experiments were simulated at mesoscale. Furthermore, Gan et al. ${ }^{[9]}$ performed numerical simulations of wedge indentation into fcc cubic single crystals. In this work the stresses, shear strains, and crystal lattice rotation map within the material were obtained and the numerical predictions were validated with the experimental results (nanoindentation tests and EBSD investigation of the indented area). Moreover, both spherical and pyramidal indentation tests in fcc single crystals were simulated by Alcala et al. ${ }^{[10]}$ and Narayanan et al. ${ }^{[11]}$ Zahedi et al.$^{[12]}$ developed their own crystal plasticity models to investigate spherical indentation in copper single crystals.

Not only $\mathrm{Cu}$ but also single crystals made of other materials have been investigated recently. The nanoscale anisotropic elastic-plastic behavior of single crystal aragonite using nanoindentation with a Berkovich probe and scanning force microscope imaging was studied by Kearney et al. ${ }^{[13]} \mathrm{Ju}$ et al. used molecular dynamic (MD) simulations to elucidate the anisotropic characteristics (pile-up patterns and atomic stress distribution) in the material responses for nickel substrates with different surface orientations. ${ }^{[14]}$ Yoshida et al. ${ }^{[15]}$ performed nanoindentation tests with a Berkovich probe on aluminum single crystals in order to investigate the effect of crystallographic planes on the yield load and the hardness of the metals in nearly perfect crystals. Britton et al. combined nanoindentation (Berkovich probe), electron backscatter diffraction (EBSD), and CPFEM simulation to examine the anisotropy in the indentation behavior of individual grains within alpha Ti-polycrystals. ${ }^{[16]}$ Xie et al. ${ }^{[17]}$ reported that in an Ni-based single crystal superalloy indented on the (001) and (011) planes, the deformation also depends on the crystallographic orientation.

The residual imprints in anisotropic (multilayer) coatings were investigated using AFM, and simple model of the elastic-plastic penetration of a Berkovich pyramid has been developed by Kravchuk et al ${ }^{[18]}$

Unfortunately, few researchers have addressed the problem of nanoindentation experiments with small loads and pyramidal tips. Therefore, the pile-up pattern observed in these experiments depends mainly on the crystal structure of the indented surface. Conversely, Chiu and Ngan ${ }^{[19,20]}$ who investigated single crystals of $\mathrm{Ni}_{3} \mathrm{Al}$, conducted nanoindentation experiments in the (111) plane using a Berkovich indenter with forces ranging from 0.8 to $8 \mathrm{mN}$. Next they investigated the impressions using transmission electron microscopy (TEM). They reported that indentation plasticity zones consist of two regions: a core with a very high dislocation density and a surrounding region where the dislocation density is much lower. They observed that the core and a surrounding low dislocation density zone did not evolve in a self-similar manner as the load increased. It was suggested that this is a new aspect of the indentation size effect (ISE) whose implications should be further investigated.

Therefore, in this paper, we present the results of nanoindentation using a Berkovich tip and very small indentation depths (down to tens of nanometres). For the experiments, we indented single crystalline copper specimens with different orientations. To imagine the sample surface, scanning force microscopy was used. We have observed that at very small indentation loads, the pile-up patterns have a random character and are closer to the tip symmetry rather than the crystal symmetry. This effect may strongly influence the results of the nanoindentation tests and significantly enhances our knowledge of the mechanical properties of the surface of single crystals.

\section{EXPERIMENTAL}

\section{A. Nanoindentation Setup}

The experiments were carried out with a CSM UltraNanoindentation Tester. The device includes an XYZ sample stage, which is used to precisely position the sample under the indenter, the atomic force microscope (AFM), or the optical microscope. Firstly, the sample is carefully positioned under the indenter tip. The measuring head consists of a measuring axis and a reference axis. Each has its own actuating means, displacement measuring system, and applied force measuring means. The indentation measurement can, therefore, be performed relative to a reference whereof the application force on the sample is accurately controlled. The independent control of the axis of reference makes it possible to prevent any disturbance of the sample and/or of the measuring instrument itself (i.e., resulting from a temperature variation). The load application is done by piezoactuator, whereas the load is measured by a capacitive sensor. The highest load resolution which can be achieved is $10 \mathrm{nN}$. Displacement is measured by a differential capacitive sensor with $0.001-\mathrm{nm}$ resolution. Typical values of the thermal drift are less than $0.05 \mathrm{~nm} /$ min. After the indentation, the sample is positioned under the AFM to imagine the sample surface. The optical microscope can also be used to investigate the sample surface, especially in the case of larger indents.

\section{B. Sample Preparation and Experiments}

A copper single crystal was drawn from the melt and cut precisely into samples by spark erosion and wire saw to give (001)-, (011)-, and (111)-oriented surface planes. The surfaces were polished, then electro-polished, and finally carefully and rigorously cleaned with distilled water and isopropyl alcohol. The indentation tests with a wide range of maximum indentation loads (from 0.1 to $8 \mathrm{mN}$ ) were performed on all the above orientations with the aim of investigating the influence of indentation depth on the surface topography after the indentation. The Berkovich indenter was used in order to determine whether the pile-up pattern depends on the indenter shape or on the crystallographic structure of the material. The load velocity was set to be equal to 50 pet of the maximum indentation load per minute. The indentation tests with the same parameters were repeated independently at least three times for the sake of statistical analysis and to avoid random inaccuracies and errors. 
The experimental procedure is virtually the same as that used by other researchers (i.e., described in References 3 or 4 ). Nevertheless, the application of small forces (down to $0.1 \mathrm{mN}$ ) and the Berkovich indenter revealed interesting properties of the pile-up patterns and, therefore, significantly expands prior work.

\section{Imprints Imaging and Data Processing}

To obtain detailed data of the imprint shape and pileup pattern, the AFM was used. After the indentation test, the imprint was precisely positioned under the AFM tip and scanned accurately. The horizontal resolution of the piezoactuators in the case of the smallest imprints was $1 \mathrm{~nm} /$ pixel, scanning speed was $0.5 \mu \mathrm{m} \mathrm{s}^{-1}$, and the signal/noise ratio was about 4 . It should be noted that due to the convolution, the real horizontal resolution was not higher than $10 \mathrm{~nm}$. The digital open feedback loop was used for small imprints imaging and the closed-feedback loop was applied, while bigger imprints were scanned. The resolution in the Zrange was $0.01 \mathrm{~nm}$. The image processing procedures embedded in the software governing the AFM were turned off, therefore, the row data of the scanned surface topography were next post-processed using the special Hommel Map ${ }^{\circledR}$ software.

To remove the general slope of the surface from AFM images, they were leveled using least-square plane method. It should be noted that the real area of imprint was excluded from the calculations. The results of operation are shown in Figure 1(a). In many figures in this paper, in order to show the pile-up patterns more in detail, the measured imprint was artificially truncated at the bottom by the thresholding operation (Figure 1(b)). It was especially useful for suppressing the deep, high load imprints, as the absolute value of their ordinates is much higher than that of the surrounding pile-ups. The rough image of the pile-up pattern was also obtained by the optical microscopy.

\section{RESULTS}

The nanoindentation tests were performed in a relatively large range of loads from 0.1 to $8 \mathrm{mN}$. The following load levels were applied: $0.1,03,0.45,0.6$, $0.8 \mathrm{mN}$ (the smallest loads), 1, 2, $3 \mathrm{mN}$ (medium loads), and $5,8 \mathrm{mN}$ (the greatest loads). Only the most characteristic results for each load level have been selected and shown in the subsequent figures to illustrate the discussion and conclusions presented in this paper.

\section{A. Description of General Pile-Up Pattern}

As outlined in the introduction, the anisotropy associated with nanoindentation of a single crystal in different crystallographic orientations has been intensively studied recently due to the fact that the pile-up and sink-in patterns are significantly different than in the case of isotropic materials. To obtain a deeper insight into the creation of indentation pile-up patterns in single crystals, many numerical simulations were performed, especially with the use of CPFEM. It is evident that for relatively large indents, according to the crystal plasticity theory, the pile-up pattern depends on the orientation of the surface plane. On the other hand, our results indicate that in the case of small indents, the shapes and spatial locations of the pile-ups which appear on the copper single crystal surfaces are significantly different.

We have observed that the formation of pile-up or sink-in depends on the applied load and on the orientation of crystal with respect to the indentation direction. The presented results also indicate that when non-axis-symmetric (Vickers or Berkovich pyramid) probes are used, the system of pile-ups and sink-ins is influenced by the orientation of the pyramid sides with respect to the crystal axes. Additionally, variation of the load induces a change in the number, direction, and height of the pile-ups generated in the indentation test.

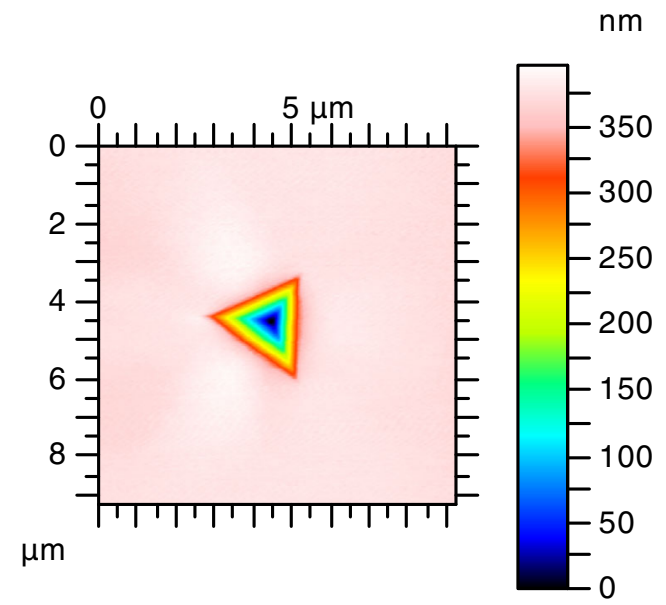

(a)

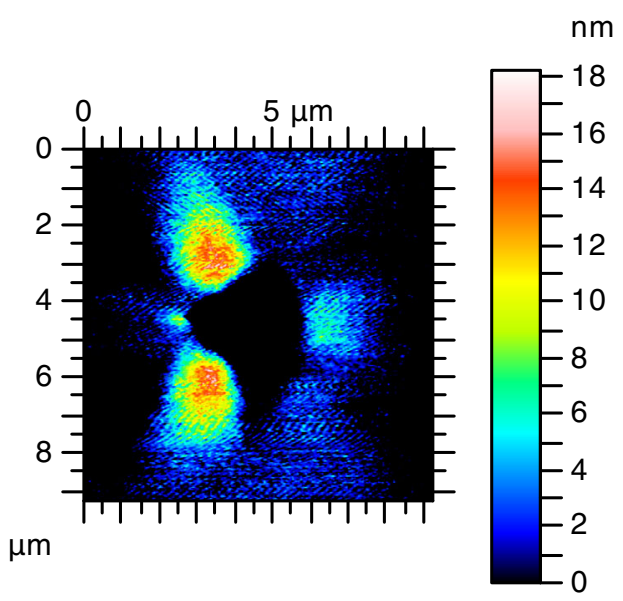

(b)

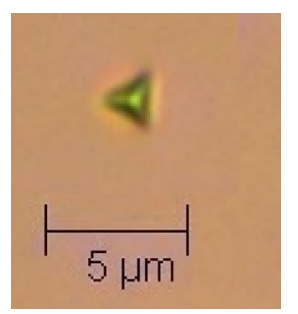

(c)

Fig. 1-Data processing. (a) Imprint image after the line by line correction. (b) The same image after the thresholding operation. (c) Optical microscope image of an imprint. 
1. Smallest loads: similar response for [001], [011] and [111] directions of indentation

In the first stage, when low loads are applied, distinct pile-ups located close to the sides of the residual imprint can be observed (Figure 2). These pile-ups have a height of about $14 \mathrm{~nm}$ for the lowest load $(0.1 \mathrm{mN})$. The pileups have a specific form: they are elongated in the direction of one side of the triangular impression. Furthermore, the fragment of hillock adjacent to the triangle is almost straight and the hillock is steeper on this side than on the other sides. Moreover, the remaining part of its circumference has a round shape. The number of pile-ups is not constant and has a rather random structure; that is, in many indentation tests performed with the lowest load $(0.1 \mathrm{mN})$, one, two, or three pile-ups occurred around the triangular impression. When the indentation load $P$ is bigger than $0.3 \mathrm{mN}$, one decidedly dominant pile-up is observed. This form and height of the pile-ups are maintained up to loads of about 0.6 to $0.8 \mathrm{mN}$. The behavior of the material in this first stage is qualitatively similar for all indentation directions. However, it is worth noting that in many performed tests, the formation of two or three distinct pile-ups takes place more frequently for the (100)-oriented surface. It is also important that the detailed analysis of the surface topography around the impressions indicates that besides distinct, relatively high pile-ups, some contours (traces) of an acclivity of the material are also visible, which will be called pseudopile-ups in the subsequent text (Figures 2, 3, and 4).
They exhibit a certain symmetry, however, and due to their very low height (a few nanometres), they should be distinguished from the 'real' pile-ups.

One can conclude that for the smallest loads, the shape and the position (symmetry) of pile-up patterns correspond to the symmetry of the tip shape. It is similar to the case of isotropic materials, for example, three hillocks occur in the case of a Berkovich tip (Figure 6) and four in the case of a Vickers tip; the only difference is that in the nanoscale the number of hillocks generated around the imprint is not repeatable and has a random character.

\section{Indentation in (111) oriented plane with medium and greatest loads}

On the other hand, in the second stage, when the load increases further, the response of the material depends on the indentation direction. When the (111)-oriented surface is indented, one observes a qualitative change of the form of each hillock; its form is closer to the axissymmetric one. Its summit moves away from the impression (contact boundary) and the whole circumference becomes more round (Figures 3, 4). In the second stage, for (111)-oriented surfaces, the number of pile-ups changes: for $P=0.8$ to $1.5 \mathrm{mN}$, two distinct pile-ups and three pseudo-pile-ups are visible (Figure 3(a)); next, for $P=3$ to $8 \mathrm{mN}$, three pile-ups dominate, and the remaining three pseudo-pile-ups are difficult to distinguish practically (Figure 4(a)). Finally, three large hillocks can be seen, Figure 5(a), two of

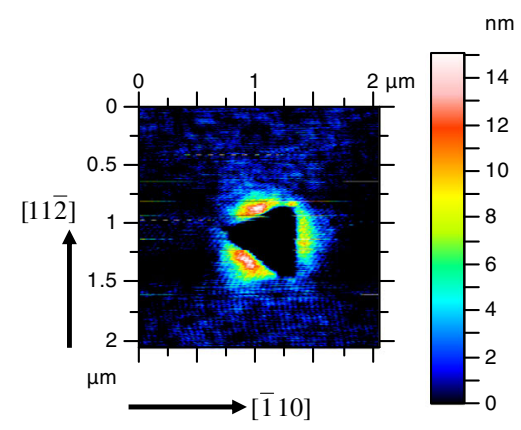

(a)

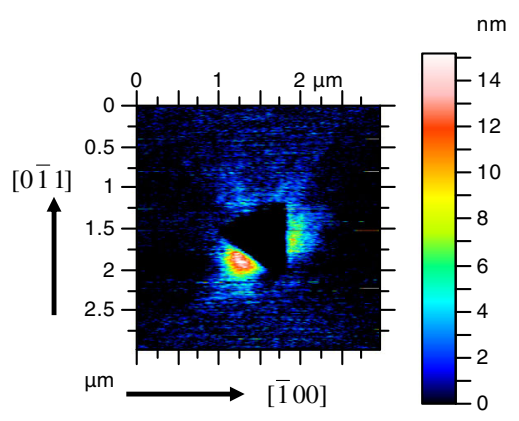

(b)

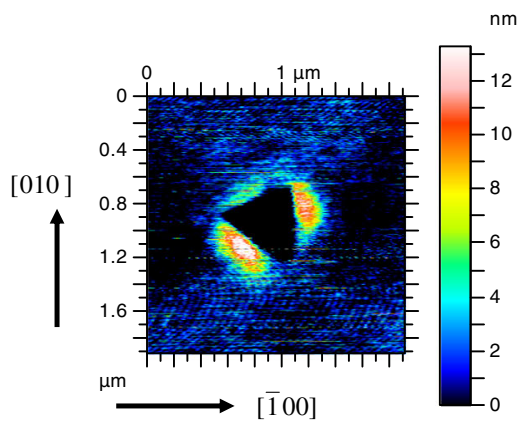

(c)

Fig. 2-Pile-up patterns for small indentation loads. (a) $0.1 \mathrm{mN}$, (111)-oriented surface; (b) $0.3 \mathrm{mN}$, (011)-oriented surface; (c) $0.1 \mathrm{mN}$, (001)-oriented surface.

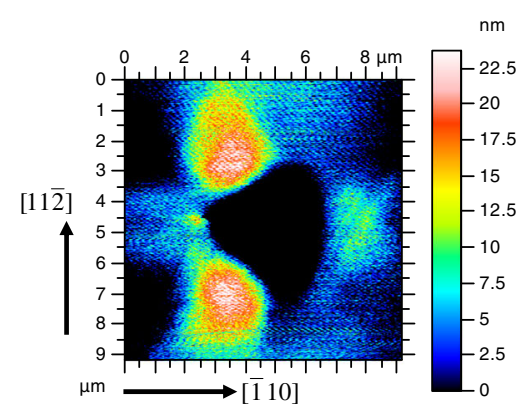

(a)

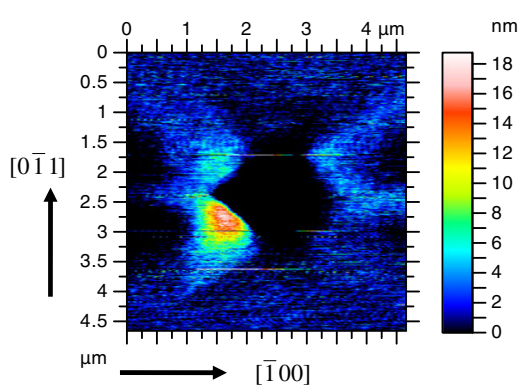

(b)

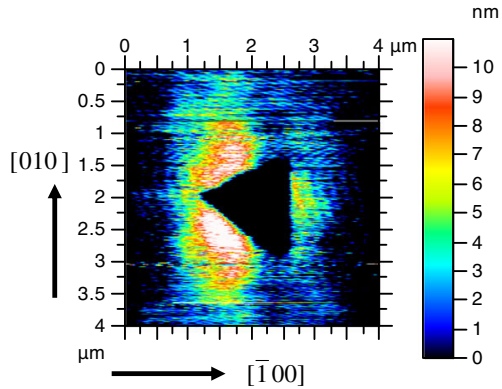

(c)

Fig. 3-Pile-up patterns for $1 \mathrm{mN}$ indentation loads. (a) (111)-oriented surface; (b) (011)-oriented surface; (c) (001)-oriented surface. 
them, near to the apex of the triangular impression and oriented in directions $[01 \overline{1}]$ and $[10 \overline{1}]$, are much higher than the third, which is close to the opposite side of the triangle and oriented in the direction [110]. The fourth, small but distinct pile-up is generated at the apex of the triangular impression ([1] $\overline{1}])$. Two pseudo-pile-ups can

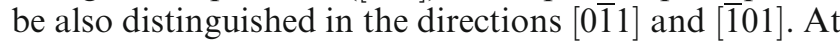
higher values of the applied load, a small sink-in occurs in the vicinity of the triangle sides, close to the boundary of the imprint. This sink-in zone has the same character of symmetry as the tip (Figure 5(a)).

\section{Indentation in (011) oriented plane with medium} and greatest loads

The behavior of material is slightly different to that described above when the load increases and the (011) plane is indented. At medium loads (1 to $1.5 \mathrm{mN}$ ), two distinct hillocks are formed in directions $[21 \overline{1}]$ and $[2 \overline{11}]$

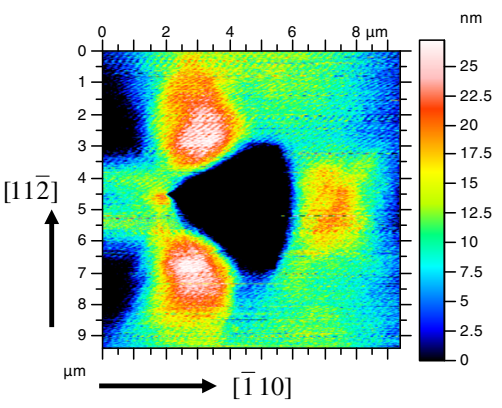

(a)

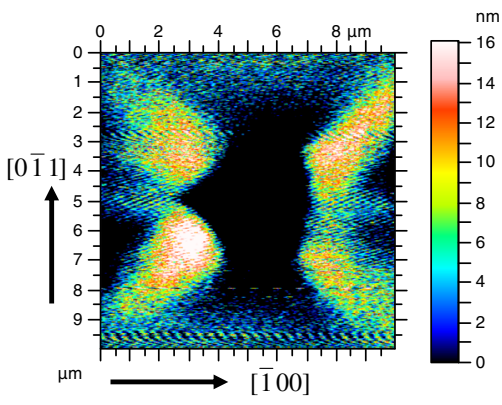

(b)

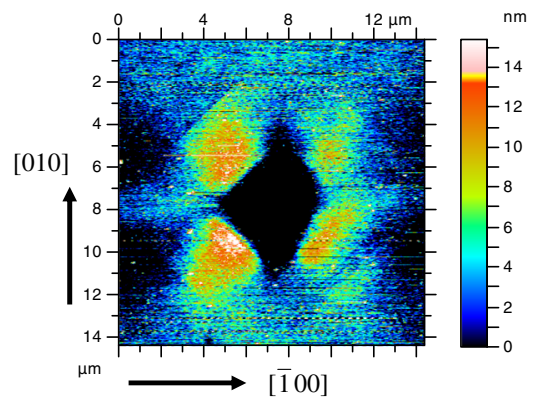

(c)

Fig. 4 -Pile-up patterns for $5 \mathrm{mN}$ indentation loads. (a) (111)-oriented surface. The pseudo-pile-ups can be seen. (b) (011)-oriented surface; (c) (001)-oriented surface.

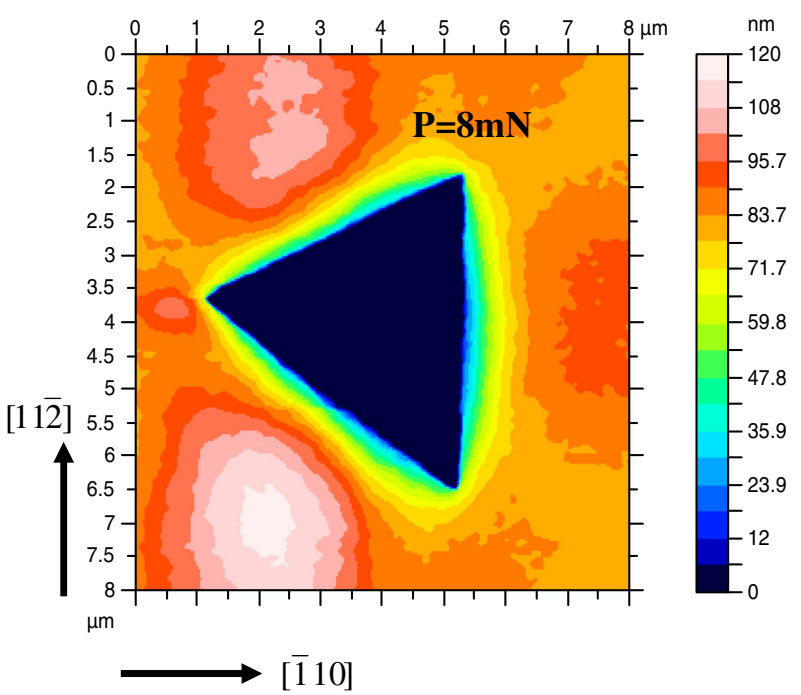

(a)

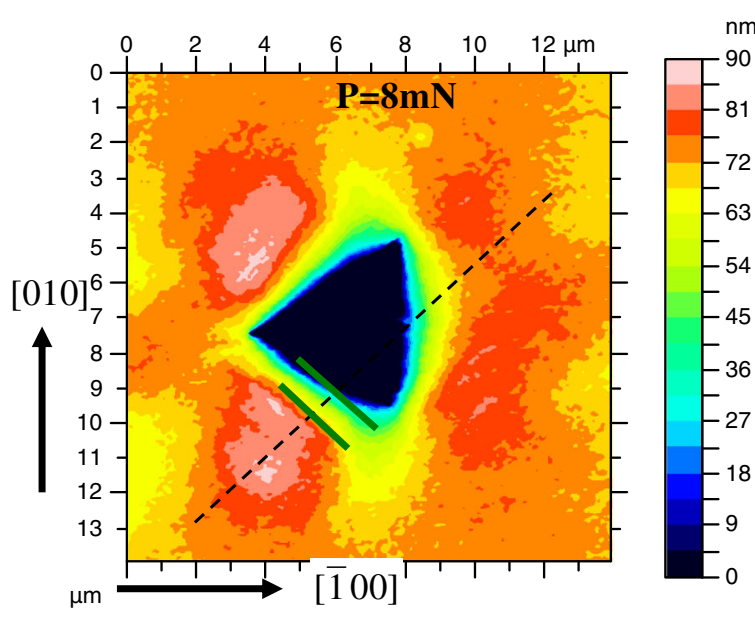

(b)

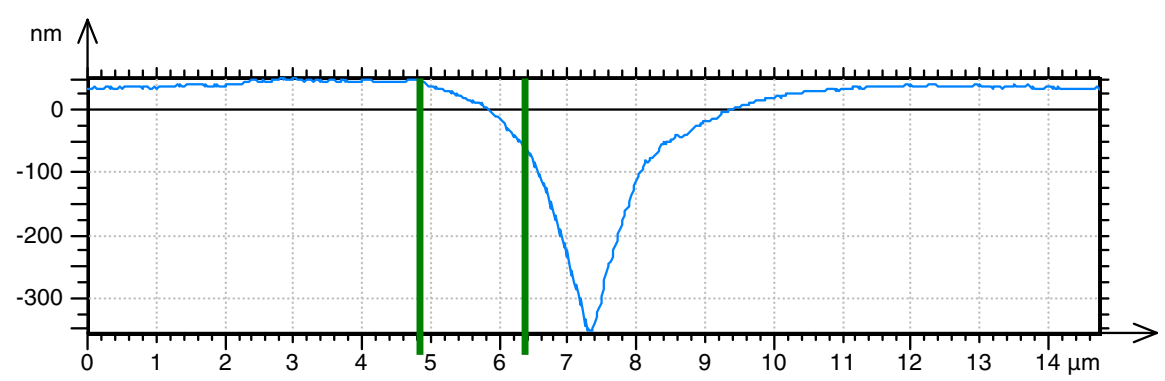

(c)

Fig. 5-Sink-in for greatest indentation loads, (001)-oriented surface. (a) (111)-oriented surface. (b) (001)-oriented surface. (c) profile from picture (b). The sink-in can be observed between the green lines (Color figure online). 
in the vicinity of the apex of the triangular impression, and two pseudo-pile-ups can be seen at the opposite side of the impression triangle (in the $[\overline{21} 1]$ and $[\overline{2} 11]$ directions) (Figure 3(b)). The elongated form of each pile-up generated at smallest loads is not preserved; its form is similar to that observed for the (111) indentation direction rather than that observed for the (100) direction; that is, the hillock is closer to the axissymmetric one and the whole circumference becomes more round and its summit moves away from the impression (contact boundary).

Once the load increases, the pairs of pile-ups and pseudo-pile-ups grow. Eventually, however, there are two pairs of hillocks: those which are located at the apex

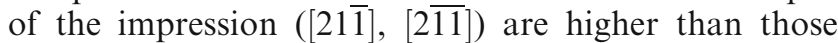
located at the opposite side ([211], [211]). The summits of the pile-ups are located at the corners of a rectangle. The sink-in zones that start to form in the vicinity of the contact boundary expand in the $[01 \overline{1}]$ and $[0 \overline{1} 1]$ directions. When the load further increases up to the greatest values, these sink-in zones build valleys that separate the higher and lower pairs of hillocks. The distance between two pairs is greater, and thus the valleys are wider than in the case of the imprint in the (001) plane (Figures 4(b) and (c)). The traces of slip planes are not visible.

\section{Indentation in (001) oriented plane with medium and greatest loads}

In the case of the (001)-oriented surface, the pile-ups generated at larger loads are qualitatively different from those observed in the case of the (111) and (011) indentation directions. They maintain their elongated shape formed at smallest loads, but they are wider and traces of slip planes can be distinguished. The number of pile-ups changes: starting from $P=1.5 \mathrm{mN}$, two distinct and high pile-ups are formed in the directions $[1 \overline{1} 0]$ and [110] close to the apex of the impression triangle (Figure 3(c)); next, when the load increases they grow, and in the direction $[0 \overline{1} 0]$ (the opposite side of the triangular impression) a new hillock appears that is further split into two more pile-ups (in the [110] and [110] directions) that are much smaller than those formed previously (Figure 4(c)). The sink-in zones that start to form in the vicinity of the contact boundary are wider than those observed for indentation in the (111) plane. When the load increases these expanding sink-in zones build valleys that separate the two higher ([1 $\overline{1} 0]$, $[110])$ and two lower $([\overline{1} 10],[\overline{110}])$ hillocks (Figure 5(b)). Finally, one observes two pairs of hillocks. The hillocks constituting the pair located at the apex of the triangular imprint are much higher than those located at the opposite side of the imprint. The summits of the pile-ups are located at the corners of a square.

Generally, at the greatest loads, the topography of pile-ups is an effect of crystal symmetry rather than the tip symmetry and the process of the pile-ups formation is more repeatable, than in the case of the smallest loads.

\section{B. The Evolution of Pile-Up Positions}

In isotropic materials indented with the Berkovich tip, the pile-ups summits occur in the directions of bisectors of the sides of the triangular impression of the pyramidal indenter (denoted as $N_{\mathrm{ti}}, i=1,2,3$; Figure 6). In the case of anisotropy, there are certain directions that are characteristic for the orientation of the indented plane. These directions, denoted as $T_{i}$, depend on the crystal symmetry; they are structurally favorable for the formation of the characteristic pile-up patterns and can be determined by the hillocks generated by means of indentation with axis-symmetric tips (spherical, conical). ${ }^{[3,4]}$ When the Berkovich tip is used, the pile-up pattern depends not only on the orientation of the indented surface but also on the orientation of the sides of the pyramidal tip, $N_{\mathrm{ti}}$, with respect to $T_{\mathrm{i}}$. A detailed study of this phenomenon reveals an interesting property of the pile-up pattern. Figure 7 shows AFM micrographs of the pile-up patterns on the (001)-, (011), and (111)-oriented surfaces after indentation with different maximum loads. The angular position of the pile-up summits is also shown. It appears that the pileup pattern around the $0.1 \mathrm{mN}$ impressions (indentation depth equals about $60 \mathrm{~nm}$ ) looks similar to the pattern around indents made in isotropic material (Figure 6). With increases in the indentation load, in the case of (100)- and (110)-oriented surfaces, the pile-ups slowly change their angular position and rotate along the axis perpendicular to the surface toward the positions observed for higher loads. These positions correspond to the crystallographic orientation of the indented surfaces. On the other hand, for the (111)-oriented surface, the rotation is not observed. As described before, new pile-ups or pseudo-pile-ups appear. This difference occurs due to the fact that, in our experiments, the tip symmetry agrees with the symmetry of the (111)-oriented surface. Therefore, these pile-ups are in their final angular positions from the beginning.

Figure 8 shows three plots in which the angular positions of the pile-up summits are indicated. The $0 \mathrm{deg}$ angle corresponds to the direction defined by the deepest point of the impression and the triangular impression's apex on the left ([010] for the $(001)$ orientation, $[01 \overline{1}]$ for the $(011)$ orientation, and $[\overline{1} 10]$ for the (111) orienta-

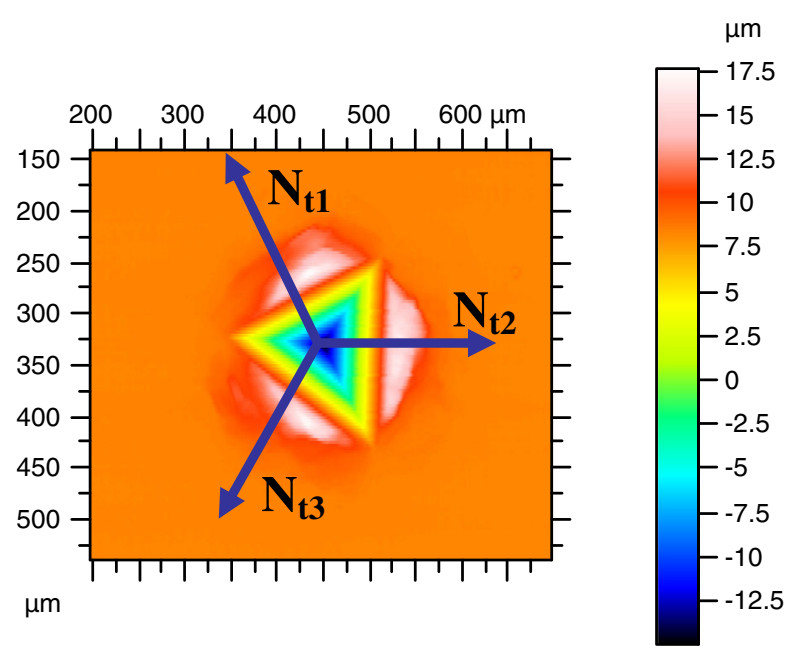

Fig. 6-Isotropic material indented with Berkovich tip. 


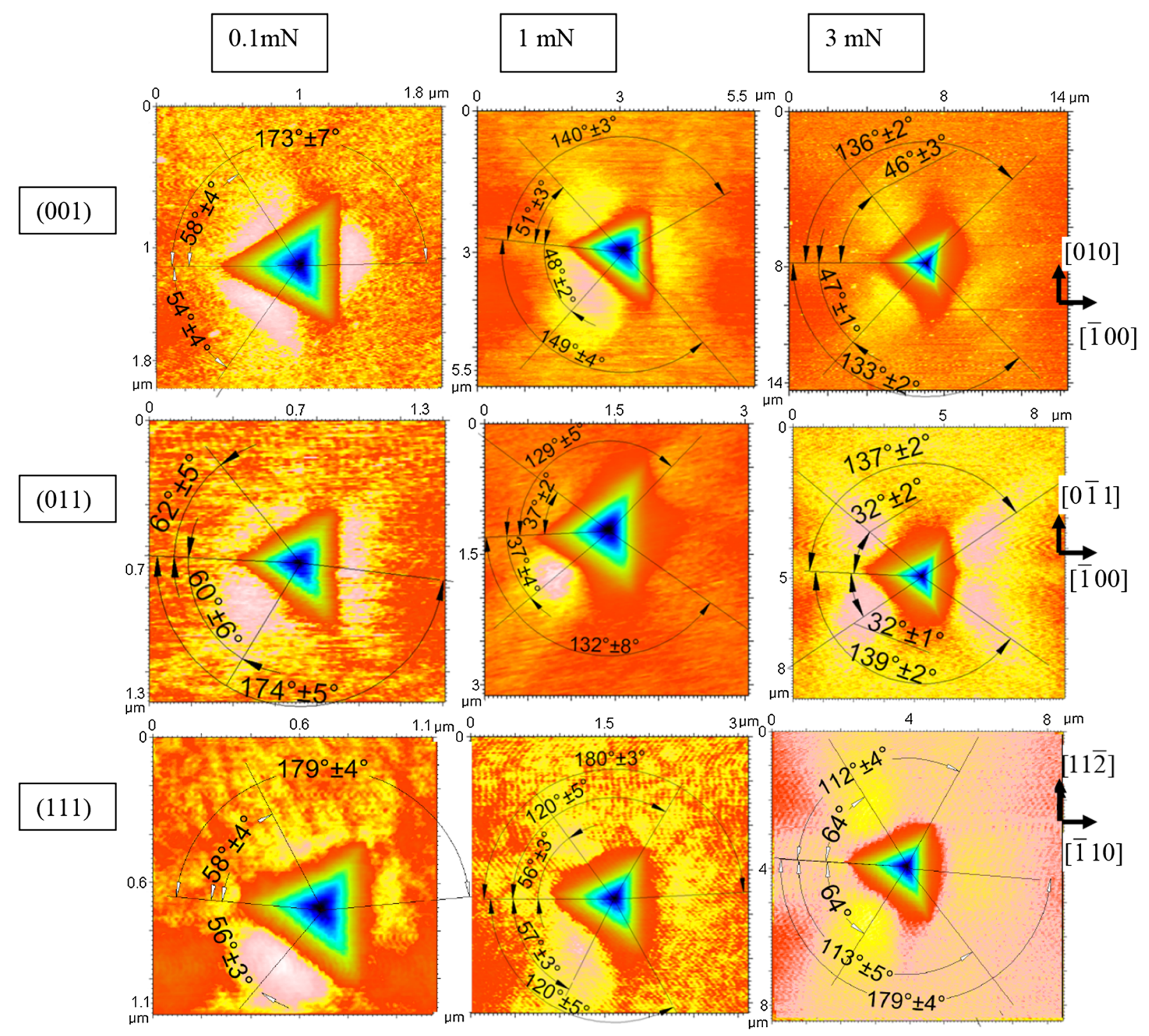

Fig. 7-AFM micrographs of the angular position changes of the pile-ups.

tion). Angles above this direction in the figure are positive and those below are negative. It is evident that, with low indentation forces, for each orientation, the pile-ups appear at three angular orientations: $60,-60$, and $180 \mathrm{deg}$. The change in the pile-ups' angular positions can be clearly seen in Figures 8(a) and (b). The constant angular position of the pile-ups on the (111)-oriented surface is also shown in Figure 8(c).

Not only the angular position of the pile-ups but also their distance from the boundary of the imprint changes with increases in the indentation load. Figure 9 shows normalized profiles of indents (both coordinates are divided by the maximal penetration depth) made with different indentation loads in the (100)-oriented surface. The depth of the deepest point of the indent equals zero. The height of the flat surface around the impression equals one. The profiles were obtained along the direction defined by the deepest point of the imprint and the summit of a pile-up. These results show the evolution of the pile-up distance from the impression's edge and the changes with the pile-up shape. At small loads (Figure 9(a)), the pile-up is close to the boundary of the imprint and is rather high compared with the penetration depth. As the indentation load increases, the normalized pile-ups become lower and their summits move away from the impression's edge. On the other hand, for the medium and the highest loads, when summits of hillocks are at a significant distance from the impression edge, the sink-in appears (Figures 5 and 9(b)) close to the contact boundary. When the greatest loads are taken into consideration (Figure 9(b)), it can be clearly seen that after normalization the profiles of pile-up patterns look very similar.

\section{The Mechanical Properties of Different Orientations}

According to the results of force-indentation depth $(P-h)$ curves obtained during the experiments, the 


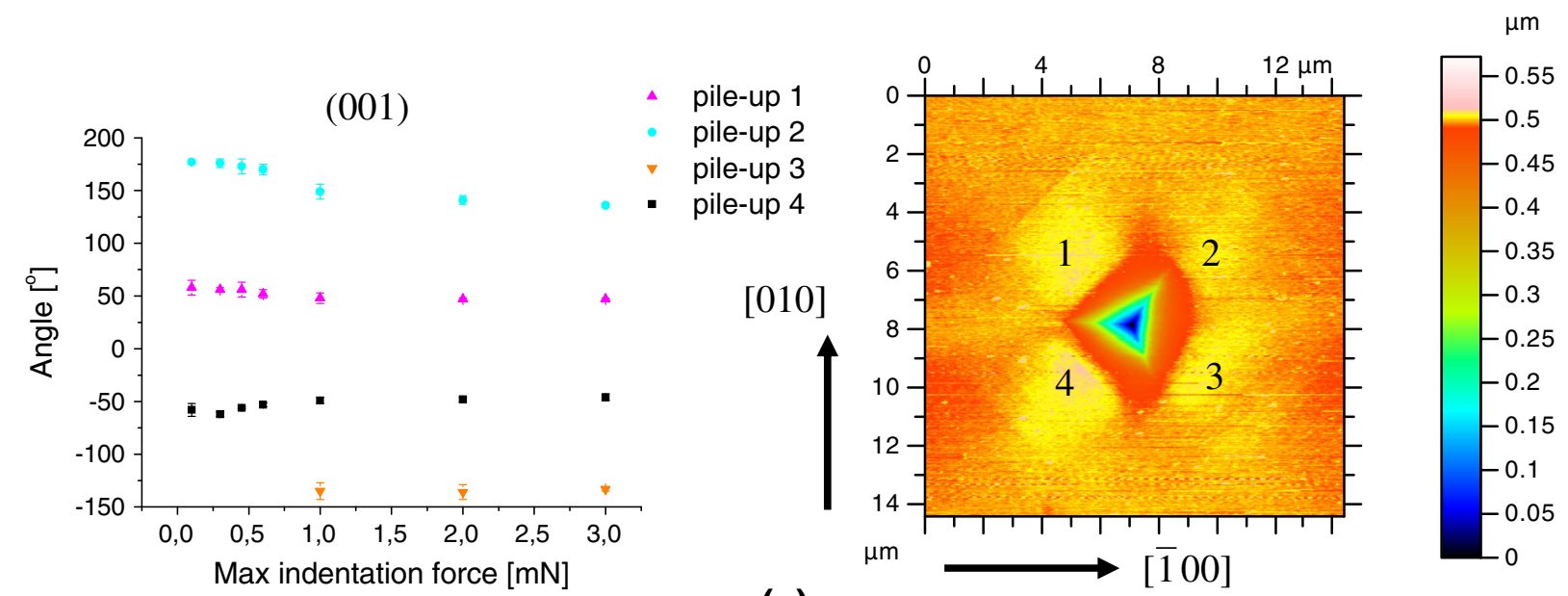

(a)
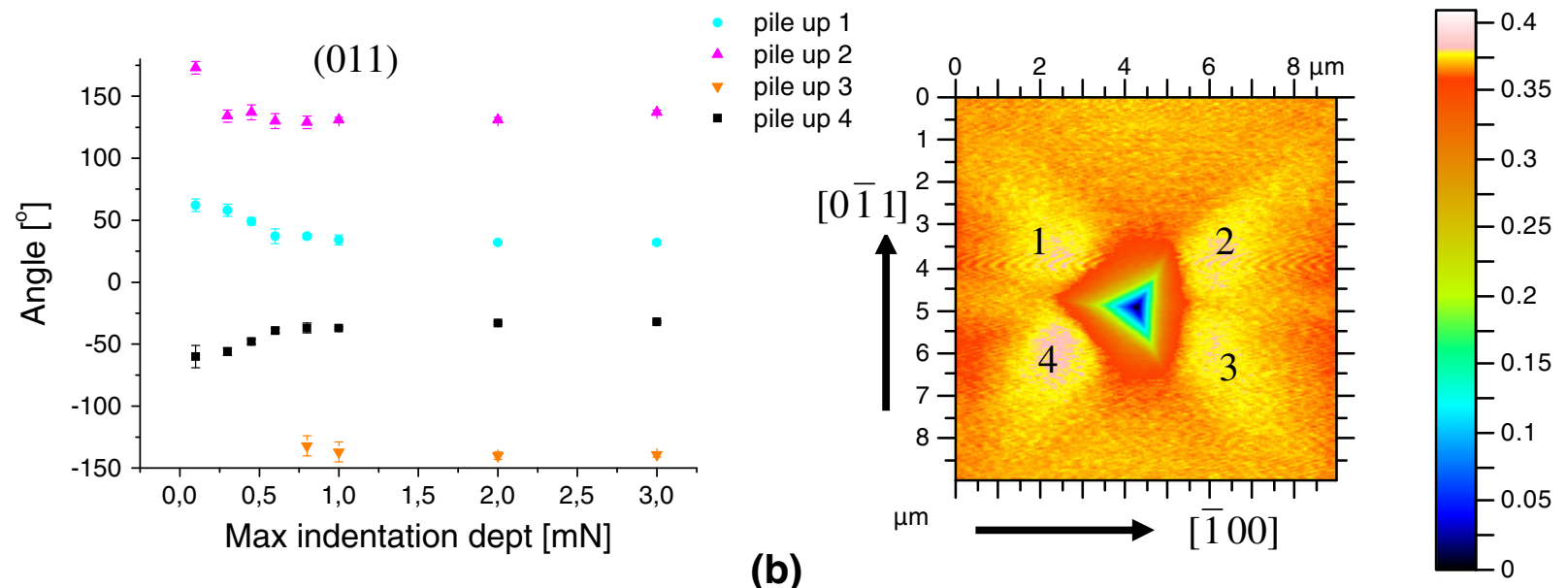

(b)
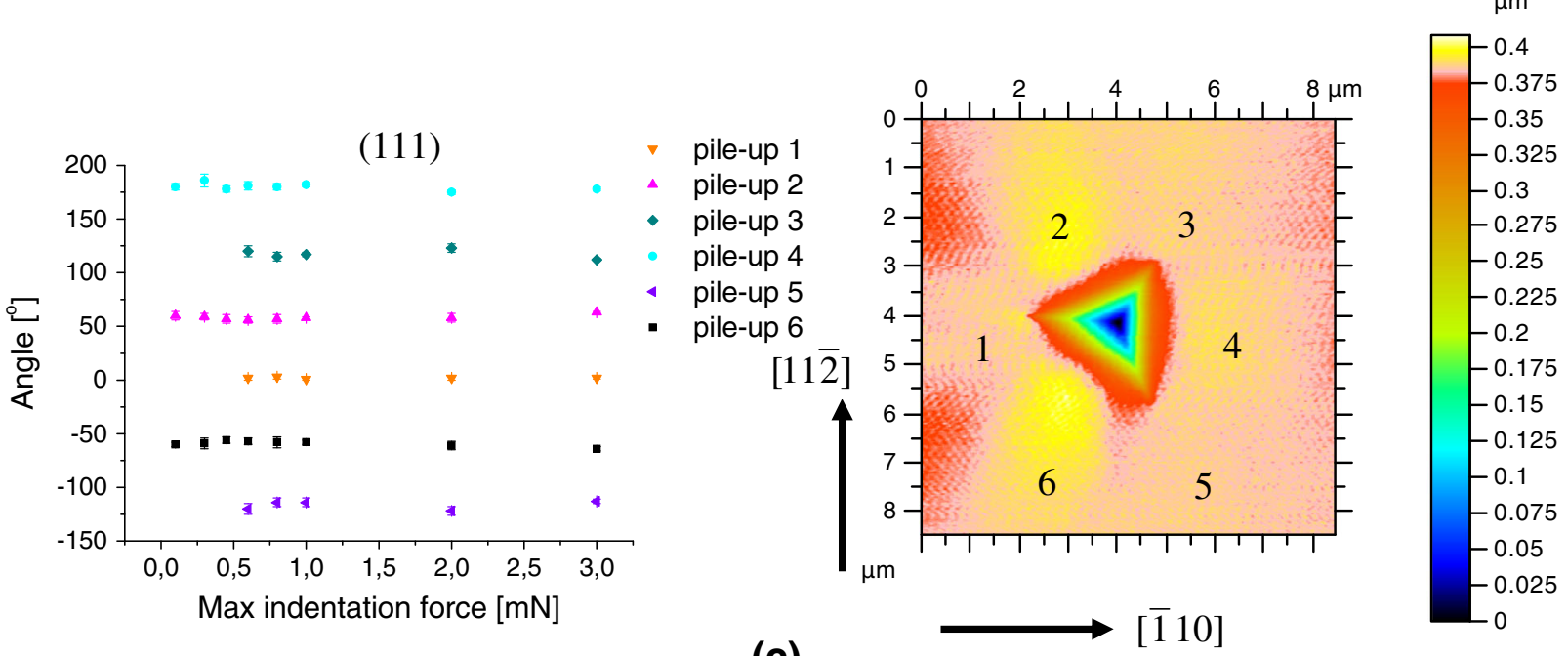

(c)

Fig. 8-Plots of the angular positions of the pile-ups.

surfaces of the copper single crystal with different orientations have slightly different mechanical properties. Figure 10(a) shows many different $P-h$ curves obtained from the indentation experiments with $0.1 \mathrm{mN}$ maximum load. For the smallest loads, the drops on $P-h$ curves can be observed that are an effect 


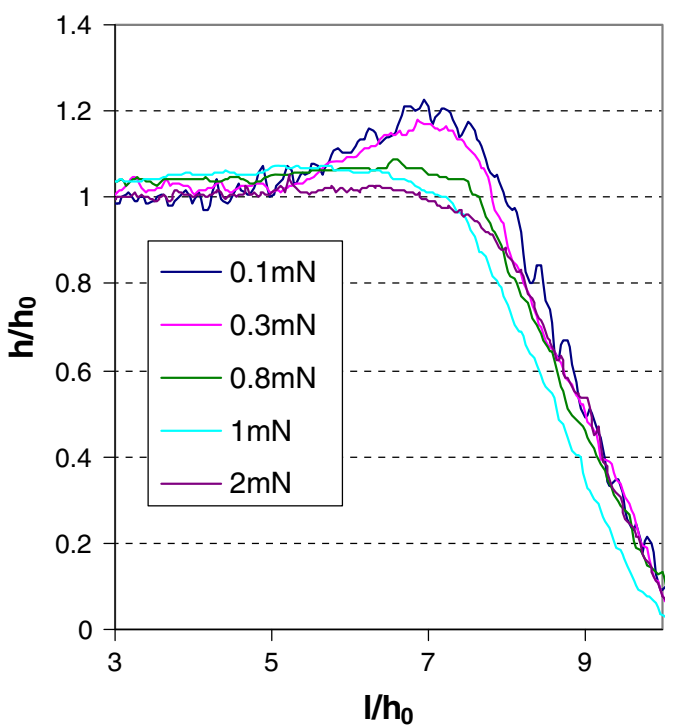

(a)

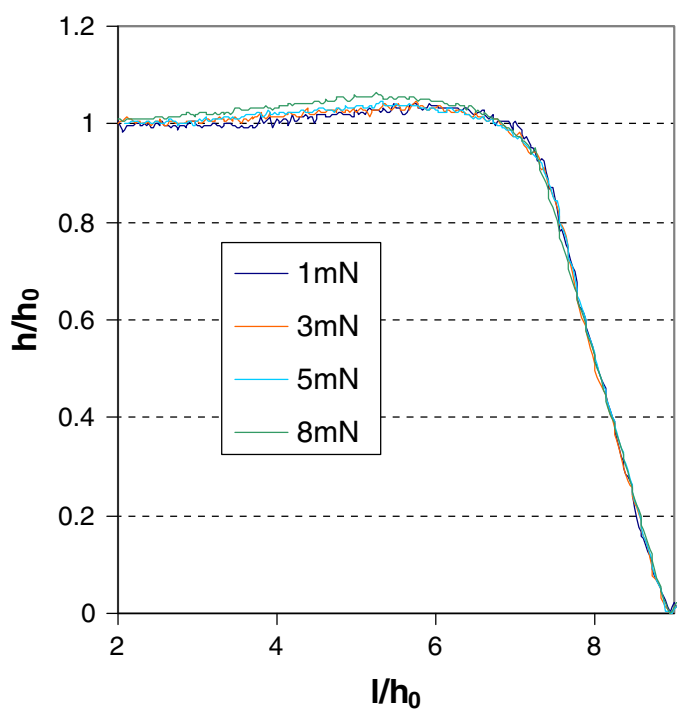

(b)

Fig. 9-Normalized profiles of indents made with different indentation loads in the (001)-oriented surface.

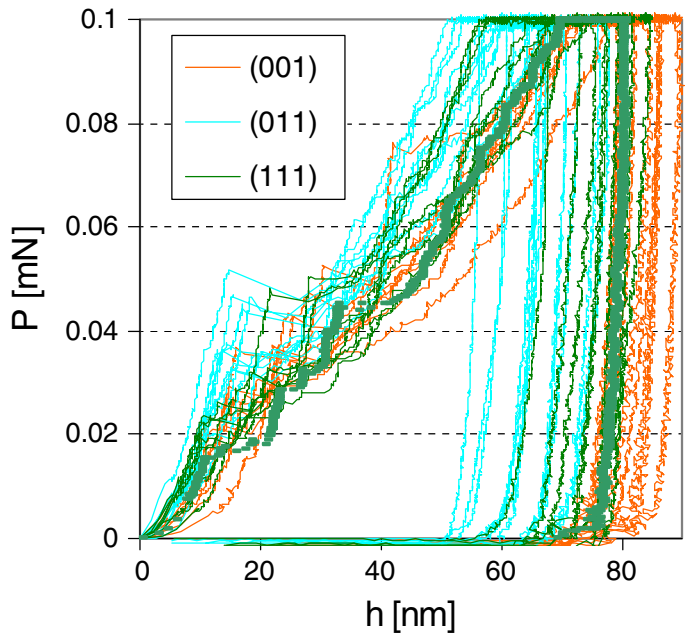

(a)

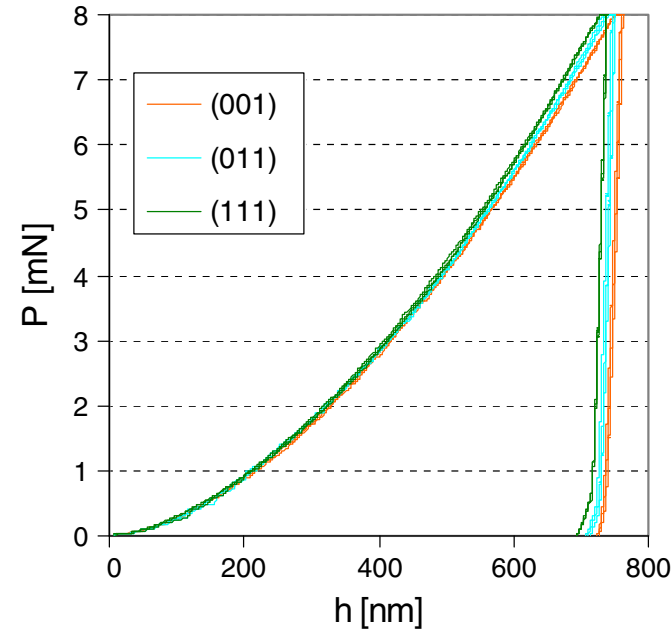

(b)

Fig. $10-P-h$ curves for 0.1 and $8 \mathrm{mN}$ indents.

of pop-in phenomena that occur in nanoindentation test of single crystals. The pop-in has been intensively studied for brittle crystals (e.g., sapphire) and in the case of single crystal copper has been observed by Kucharski et al.,${ }^{[1]}$ however, as in Reference 21 a 'blunt' spherical tip $(R=5 \mu \mathrm{m})$ was used, the load corresponding to this phenomena was greater (about $1.5 \mathrm{mN}$ ).

It is evident that the (011)-oriented surface has the steepest loading curve, whereas the (001)-oriented surface is the most compliant surface. Hence, it is apparent that different orientations have different stiffnesses. This occurs in spite of the fact that every pile-up pattern for all orientations, at small loads, is similar to the pile-up pattern of the indentation in the isotropic material. On the other hand, when the greatest loads are investigated, the (011) and (111) orientations swap positions (Figure 10(b)). The (001)-oriented surface is still the weakest, but the strongest one is now the (111)-oriented surface. The latter relation has been also reported for spherical nanoindentation tests. ${ }^{[11]}$

\section{The Shape of the Tip}

Our results indicate that the residual nanoindentation imprints on $\mathrm{Cu}$ single crystal are not self-similar with load. The patterns evolve and in the case of small loads they are different from the patterns predicted by crystal plasticity theory. They look similar to the patterns created in an isotropic material. Therefore, it was necessary to investigate, if some small defects at the top of the tip (such as tip roundness) are not influencing 


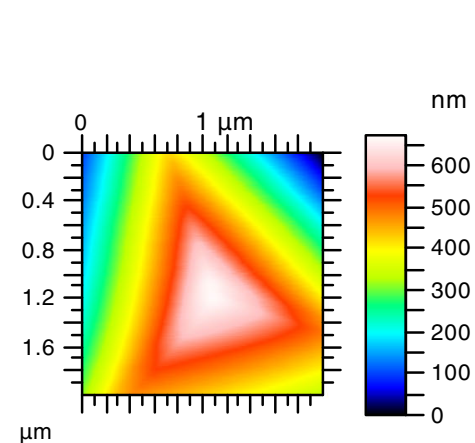

(a)

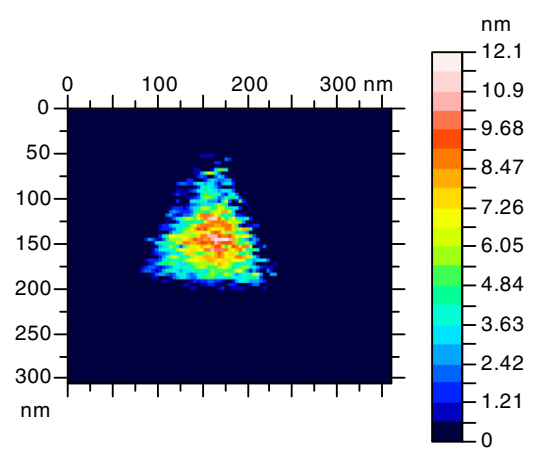

(b)

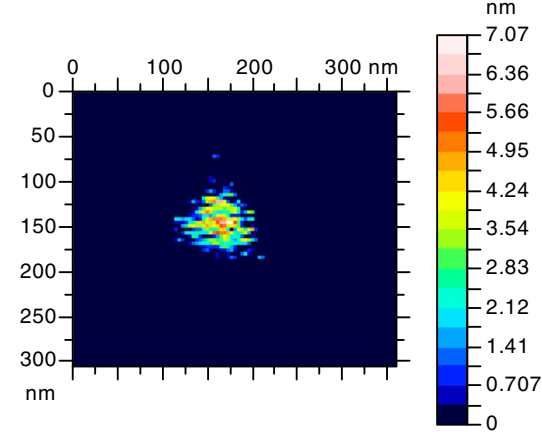

(c)

Fig. 11-AFM micrographs of the used Berkovich tip. $(a)$ in the microscale for 600 nm penetration depth; $(b)$ in the nanoscale for $12-$ nm penetration depth; $(c)$ in the nanoscale for 7-nm depth.

our measurement. We have carefully imagined the applied Berkovich tip with the highest possible resolution with the intention of evaluating the transition of the tip shape from round to pyramidal. It can be clearly seen in Figure 11 that the cross-section of the tip at $10 \mathrm{~nm}$ from the top has still the triangular shape with distinct edges. Significant deviation from the pyramidal shape can be observed at about $7 \mathrm{~nm}$ from the top of the tip. It is evident that so small deviation from the perfect pyramidal shape cannot influence the shape of pile-ups generated in our experiments. It should be noted that the unexpected, isotropic-like pile-ups are observed even in the case of imprints which depth is higher than $100 \mathrm{~nm}$. Furthermore, the tip roundness is not able to create triangular pile-up pattern, which was observed.

\section{DISCUSSION}

The results presented in this work are essentially in good agreement, in the case of bigger indents, with the results achieved by other researchers. As anticipated, the pile-up patterns which were measured in our experiments with bigger loads are very similar to those observed in the earlier studies. The position of pile-up patterns is determined by the symmetry of primary slip systems; that is, they are a result of the material translation along the intersection of the primary slip systems with the indented surface. ${ }^{[3]}$ Nevertheless, a closer look at the topography after the indentation tests revealed new observations. For example, the pile-up patterns generated when the (001) direction is indented have approximately rectangular shape, and traces of slip planes are visible. Thus, they are qualitatively different from those generated for other directions (111), (011), where the hillocks have rather circular shape. This shape can result from the activation of both primary and secondary slip systems when the other directions are indented. The sink-ins appear between the indent edge and the pile-ups (Figures 5 and 9). In the case of the (100) indented surface, when the load increases, the sink-in patterns also extend to the area lying between the generated hillocks. To explain this phenomenon, further investigation and more accurate identification of the parameters that occur in existing crystal plasticity models or the development of more advanced models are necessary. It is particularly interesting because in previous work, this effect was not observed. The pile-ups achieved the maximal height at the contact boundary. ${ }^{[3,4,11]}$ The occurrence of sink-in patterns in the vicinity of pile-up patterns was not reported in experiments and simulations. The single crystal is a material with considerable strain hardening reserve, and thus in analogy to the response of isotropic materials, one can expect the sink-in patterns to occur around the indent. Because only pile-up patterns have been observed in many previous works, the problem of the proportion of pile-up and sink-in patterns has been extensively discussed in a paper by Wang et al. ${ }^{[3]}$ The authors explained that activation of primary slip systems might lead to rather small local strain hardening; however, finally they carefully stated that the analysis of the dominant crystallographic slips serves essentially to explain the deformation patterns and their in-plane symmetry rather than the actual pile-up vs sink-in behavior.

On the basis of their numerical study of the nanoindentation of copper single crystals, Narayanan et al. ${ }^{[11]}$ presented a similar conclusion: that all orientations should exhibit pile-up patterns instead of sink-ins due to the fact that the primary slip systems activated for each orientation during nanoindentation lead to a local strain-hardening rate around the indents. According to them, this locally dominating plastic slip rate leads only to pile-up patterns. Similarly, Liu et al. ${ }^{[4]}$ did not observe sink-in patterns in their numerical and experimental investigations of single crystal copper. The solution to the problem is not evident. In an extensive study of spherical indentation in single crystal copper in the (111) direction by Zaafarani et al., ${ }^{[5,6]}$ only the angular position (sixfold symmetry) of the numerical and experimental pile-up pattern was compared. However, in Reference, 5 in the simulated sections of residual imprints, one can observe a sink-in in the vicinity of the contact boundary, while in Reference 6 where more advanced crystal plasticity theory was applied, the highest points of pile-ups are adjacent to the contact boundary. The presence of sink-in patterns has not been reported. 
As mentioned previously, the results from the nanoindentation experiments with the smallest loads are significantly different, both qualitatively and quantitatively. It is apparent that the pile-up patterns acquired in this case depend strongly on the tip symmetry (Figure 2). We tentatively attribute this effect to the random dislocation distribution close to the indented surface. According to Chiu and $\mathrm{Ngan},{ }^{[19]}$ the central densely dislocated zone at small loads is significantly larger than the contact area and the dislocations are curvy. This 'messy' dislocation structure and the dislocations' movement at the first stage of the indentation can strongly affect and distort the crystal structure of atoms close to the surface. Therefore, the atoms do not move along the crystal slip directions but move randomly, rather like grains in isotropic materials indented at macroscale (Figure 6). Similarly, Wo and $\mathrm{Ngan}^{[20]}$ observed that, despite the sharp triangular tip (Berkovich), at low loads the densely dislocated indent core had a circular shape, while at larger loads it followed the crystallographic direction of the octahedral slip systems. Furthermore, Rester et al. ${ }^{[22]}$ found that the plastically deformed zone below nano-, micro-, and macro-indentations is not self-similar. However, they have applied electron microscopy technique in their research and they cannot have observed the topography of surface in a vicinity of imprint. Furthermore, they have investigated imprints of the depth down to $250 \mathrm{~nm}$, i.e., much deeper than that analyzed in this paper. Nevertheless, their revelations may be useful to explain the effect described in this work.

Moreover, from the differences in $P-h$ curves for 0.1 and $8 \mathrm{mN}$ indents (Figure 10), it could be concluded that two different effects are observed. The fact that a random number and height of the pile-ups and a scatter of load-penetration curves occur for small indents can also support this hypothesis. According to the authors knowledge, this random character of pile-ups pattern is not an effect of the indented surface roughness, as the roughness is much smaller than the generated pile-ups, cf. Figure 9. Nevertheless, it also indicates that the material does not behave exactly like isotropic materials. Therefore, it is some kind of pseudo-isotropic behavior. It reveals a new aspect of the indentation size effect. Hence, it is clear that much additional work is required before a complete understanding can be reached. Unfortunately, a material model to account for this effect has probably not been evaluated to date. Another open question is the criteria for transition between the two stages of material response at the nanoscale, described above.

Finally, the data presented in this paper may be useful to find the answer to the question about the symmetry of the pile-up patterns in the case of the (111)-oriented surface of copper single crystal. Wang et al. ${ }^{[3]}$ and Zaafarani et al. ${ }^{[5,6]}$ claim that the sixfold symmetry should be observed, whereas Liu et al. ${ }^{[4]}$ and Narayanan et $a .^{[11]}$ maintain that there is threefold symmetry. In this paper, we have introduced the concept of 'pseudopile-up,' which is less distinct than real pile-up, and perhaps the formation of the pseudo-pile-ups could be responsible for this disagreement. The detailed theory of this effect should also be developed in future work.

\section{CONCLUSIONS}

To the knowledge of the authors, the data presented in this paper are the first of their kind. The evolution of pile-up patterns obtained in the nanoindentation experiments with different applied loads has not yet been reported. We have confirmed that for a Berkovich indenter and maximal applied load bigger than $2 \mathrm{mN}$, the distributions of out-of-plane displacements in three crystallographic orientations-(100), (011), and (111) - show pile-ups with a topographical pattern of fourfold, twofold, and sixfold (or threefold if we omit the pseudo-pile-ups) symmetry, respectively. This agrees with crystal plasticity theory and with many previous experiments. However, a detailed analysis of residual impressions with maximal applied load equal to $2 \mathrm{mN}$ and bigger reveals that both pile-up and sink-in patterns are present. In this case, the highest pile-up points were observed at a significant distance from the contact boundary. The evolution of the angular position of the pile-ups was also observed.

On the other hand, for applied loads equal to $0.45 \mathrm{mN}$ and smaller, the pile-up patterns do not depend on the crystallographic orientation of the indented surface but rather on the tip's geometry. The mechanical properties of the surfaces, however, still depend on the orientation. All these effects, which have probably not yet been presented in the literature, are encouraging and should be explored further.

\section{ACKNOWLEDGMENTS}

The present research was partially supported by the Polish National Science Centre, Grant NN 5010672 40 .

\section{OPEN ACCESS}

This article is distributed under the terms of the Creative Commons Attribution License which permits any use, distribution, and reproduction in any medium, provided the original author(s) and the source are credited.

\section{REFERENCES}

1. M.A.G. Maneiro and J. Rodriguez: Scripta Mater., 2005, vol. 52, pp. 593-98.

2. A.B. Vangroenou and S.E. Kadijk: Acta Metall., 1989, vol. 37, pp. 2613-24.

3. Y. Wang, D. Raabe, C. Kluber, and F. Roters: Acta Mater., 2004, vol. 52, pp. 2229-38.

4. Y. Liu, S. Varghese, J. Ma, M. Yoshino, H. Lu, and R. Komanduri: Int. J. Plast., 2008, vol. 24, pp. 1990-2015. 
5. N. Zaafarani, D. Raabe, R.N. Singh, F. Roters, and S. Zaefferer: Acta Mater., 2006, vol. 54, pp. 1863-76.

6. N. Zaafarani, D. Raabe, F. Roters, and S. Zaefferer: Acta Mater., 2008, vol. 56, pp. 31-42.

7. O. Casals and S. Forest: Comput. Mater. Sci., 2009, vol. 45, pp. $774-82$.

8. O. Casals, J. Ocenasek, and J. Alcala: Acta Mater., 2007, vol. 55, pp. 55-68.

9. Y.X. Gan, X. Chen, and M.H. Zhao: J. Mech. Mater. Struct., 2008, vol. 3, pp. 1429-45.

10. J. Alcala, D. Esque-de los Ojos, and J. Ocenasek: Philos. Mag., 2011, vol. 91, pp. 1050-69.

11. K.R. Narayanan, S. Subbiah, and I. Sridhar: Appl. Phys. A Mater., 2011, vol. 105, pp. 453-61.

12. A. Zahedi, M. Demiral, A. Roy, V. Babitsky, and V. Silberschmidt: Adv. Mater. Struct. IV, 2012, vol. 188, pp. 219-25.

13. C. Kearney, Z. Zhao, B.J.F. Bruet, R. Radovitzky, M.C. Boyce, and C. Ortiz: Phys. Rev. Lett., 2006, vol. 96, pp. 255505-1-5-4.
14. S.P. Ju, C.T. Wang, C.H. Chien, J.C. Huang, and S.R. Jian: Mol. Simul., 2007, vol. 33, pp. 905-17.

15. M. Yoshida, T. Sumomogi, T. Kino, M. Okumiya, and Y. Tsunekawa: J. Jpn Inst. Met., 2009, vol. 73, pp. 95-102.

16. T.B. Britton, H. Liang, F.P.E. Dunne, and A.J. Wilkinson: Proc. R. Soc. Lond. A, 2010, vol. 466, pp. 695-719.

17. G. Xie, L. Wang, J. Zhang, and L.H. Lou: Scripta Mater., 2012, vol. 66 , pp. $378-81$

18. A. Kravchuk, Z. Rymuza, and D. Jarząbek: Int. J. Mater. Res., 2009, vol. 100 (2009), pp. 933-35.

19. Y.L. Chiu and A.H.W. Ngan: Acta Mater., 2002, vol. 50, pp. 2677-91.

20. P.C. Wo, A.H.W. Ngan, and Y.L. Chiu: Scripta Mater., vol. 55, pp. $557-60$.

21. S. Kucharski, S. Stupkiewicz, and H. Petryk: Exp. Mech., DOI: 10.1007/s11340-014-9883-1.

22. M. Rester, C. Motz, and R. Pippan: Scripta Mater., 2008, vol. 59, pp. $742-45$. 